\title{
Interim Estimates of COVID-19 Vaccine Effectiveness Against COVID-19-Associated Emergency Department or Urgent Care Clinic Encounters and Hospitalizations Among Adults During SARS-CoV-2 B.1.617.2 (Delta) Variant Predominance - Nine States, June-August 2021
}

\author{
Shaun J. Grannis, $\mathrm{MD}^{1}$; Elizabeth A. Rowley, $\mathrm{DrPH}^{2}$; Toan C. Ong, $\mathrm{PhD}^{3}$; Edward Stenehjem, $\mathrm{MD}^{4}$; Nicola P. Klein, $\mathrm{MD}$, PhD 5 ; \\ Malini B. DeSilva, $\mathrm{MD}^{6}$; Allison L. Naleway, $\mathrm{PhD}^{7}$; Karthik Natarajan, $\mathrm{PhD}^{8}$; Mark G. Thompson, $\mathrm{PhD}^{9}$; VISION Network
}

On September 10, 2021, this report was posted as an MMWR Early Release on the MMWR website (https://www.cdc.gov/mmwr).

Data on COVID-19 vaccine effectiveness (VE) since the B.1.617.2 (Delta) variant of SARS-CoV-2, the virus that causes COVID-19, became the predominant circulating strain in the United States are limited (1-3). CDC used the VISION Network* to examine medical encounters $(32,867)$ from 187 hospitals and 221 emergency departments (EDs) and urgent care (UC) clinics across nine states during June-August 2021, beginning on the date the Delta variant accounted for $>50 \%$ of sequenced isolates in each medical facility's state. VISION Network methods have been published (4).

Eligible medical encounters were defined as those among adults aged $\geq 18$ years who had received SARS-CoV-2 molecular testing (primarily reverse transcription-polymerase chain reaction assay within 14 days before or 72 hours after the admission or encounter) and a COVID-19-like illness discharge diagnosis. Vaccination status was documented in electronic health records and immunization registries. Full vaccination was defined as receipt of the second dose of BNT162b2 (Pfizer-BioNTech) or mRNA-1273 (Moderna) mRNA vaccines, or a single dose of Ad26.COV2 (Janssen [Johnson \& Johnson]) vaccine $\geq 14$-days before the testing or encounter date. Patients who had received no COVID-19 vaccine doses were considered unvaccinated. Patients who had received $1 \mathrm{mRNA}$ dose only or had received the second dose $<14$ days before testing or encounter date were excluded. VE was estimated using a test-negative design, calculating the odds of receiving a positive SARS-CoV-2 test result comparing fully vaccinated and unvaccinated patients (referent group). VE was adjusted for age, geographic region, calendar time (days from January 1 to medical event), and virus circulation, and weighted for inverse propensity to be vaccinated or unvaccinated (calculated separately for each VE model). VE estimates with 95\% confidence intervals (CIs) that did not overlap were considered statistically different. This activity

\footnotetext{
* Funded by CDC, the VISION network includes Columbia University Irving Medical Center (New York), HealthPartners (Minnesota and Wisconsin), Intermountain Healthcare (Utah), Kaiser Permanente Northern California (California), Kaiser Permanente Northwest (Oregon and Washington), Regenstrief Institute (Indiana), and University of Colorado (Colorado).
}

was reviewed by CDC and was conducted consistent with applicable federal law and CDC policy. ${ }^{\dagger}$

Among fully vaccinated patients, the proportion who had received each vaccine product among hospitalizations and ED/UC encounters, respectively, were Pfizer-BioNTech, $55.3 \%$ and $53.6 \%$; Moderna, $38.8 \%$ and $36.1 \%$; and Janssen, $6.0 \%$ and $10.3 \%$. The median interval from becoming fully vaccinated to the hospital admission or $\mathrm{ED} / \mathrm{UC}$ encounter, respectively, were 110 and 93 days (Pfizer-BioNTech), 106 and 96 days (Moderna), and 94 and 94 days (Janssen).

Among adults hospitalized with COVID-19-like illness $(14,636$; median patient age $=65$ years, interquartile range $[\mathrm{IQR}]=48-77$ years), laboratory-confirmed SARS-CoV-2 infections were identified among 18.9\% (1,316 of 6,960$)$ of unvaccinated and $3.1 \%(235$ of 7,676$)$ of fully vaccinated patients. Overall, VE against COVID-19 hospitalization was $86 \%(95 \% \mathrm{CI}=82 \%-89 \%)$. VE was significantly lower among adults aged $\geq 75$ years $(76 \%)$ than among those aged $18-74$ years $(89 \%)$ (Table). The difference in VE point estimates between age groups was similar for Pfizer-BioNTech and Moderna vaccines. Across all ages, VE was significantly higher among Moderna vaccine recipients (95\%) than among Pfizer-BioNTech (80\%) or Janssen (60\%) vaccine recipients.

Among adults with ED/UC encounters for COVID-19-like illness $(18,231$; median patient age $=43$ years, IQR = 29-62 years), laboratory-confirmed SARS-CoV-2 infections were identified among $28.9 \%(3,145$ of 10,872$)$ of unvaccinated and $7.0 \%(512$ of 7,359$)$ of fully vaccinated patients. VE against COVID-19 ED/UC encounters was 82\% $(95 \% \mathrm{CI}=81 \%-84 \%)$. VE was highest among Moderna vaccine recipients $(92 \%)$, followed by Pfizer-BioNTech vaccine recipients $(77 \%)$, and was lowest $(65 \%)$ for Janssen vaccine recipients (Table).

In this multistate interim analysis of 32,867 medical encounters among adults of all ages during June-August 2021, when the Delta variant was predominant in the United States, VE of all three authorized COVID-19 vaccines combined remained high against hospitalization (86\%) and ED/UC encounters $(82 \%)$. These overall VE estimates were similar to those during

$\dagger 45$ C.F.R. part 46; 21 C.F.R. part 56. 
TABLE. COVID-19 vaccine effectiveness* against laboratory-confirmed COVID-19-associated emergency department and urgent care clinic encounters and hospitalizations ${ }^{\dagger}$ among adults during SARS-CoV-2 B.1.617.2 (Delta) variant predominance, ${ }^{\S}$ by outcome, age group, and vaccine - nine states," June-August 2021

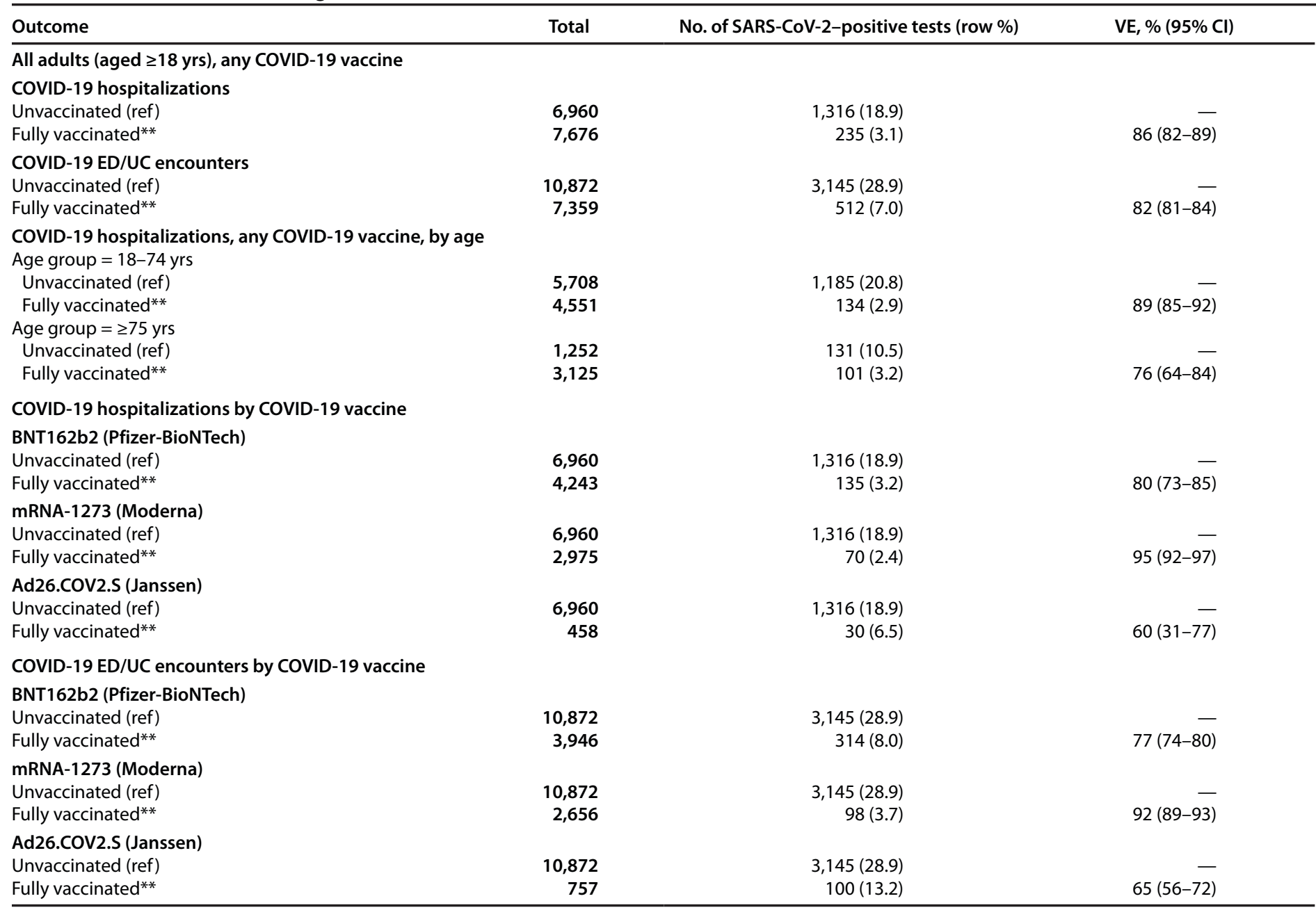

Abbreviations: $\mathrm{Cl}$ = confidence interval; $\mathrm{ED}=$ emergency department; HHS = U.S. Department of Health and Human Services; Janssen = Johnson \& Johnson vaccine; Ref $=$ referent group; $U C=$ urgent care; $V E=$ vaccine effectiveness.

* VE was estimated using a test-negative design, adjusted for age, geographic region, calendar time (cubic spline with quartile knots), and virus circulation (percentage of SARS-CoV-2-positive results from testing within the counties surrounding the facility on the date of the event) and weighted for inverse propensity to be vaccinated or unvaccinated (calculated separately for each of the $10 \mathrm{VE}$ models) using facility characteristics, sociodemographics, and underlying medical conditions.

† Medical events with a discharge code consistent with COVID-19-like illness were included, such as acute respiratory illness (e.g., COVID-19, respiratory failure, or pneumonia) or related signs or symptoms (cough, fever, dyspnea, vomiting, or diarrhea) using diagnosis codes from the ninth and tenth revisions of the International Classification of Diseases. Clinician-ordered molecular assays (e.g., real-time reverse transcription-polymerase chain reaction) for SARS-CoV-2 occurring $\leq 14$ days before to $<72$ hours after hospital admission or ED/UC encounter were included.

$\S$ Medical events occurred when Delta variant was predominant $(>50 \%)$ in each state, according to data from network partners, CDC COVID data tracker (https://covid.cdc. gov), and HHS Protects Public Data Hub (https://protect-public.hhs.gov).

9 Partners contributing both ED/UC encounters and hospitalizations in 2021 were in Indiana (range of earliest to latest medical event: July 3-26), Oregon and Washington (June 30-August 4), and Utah (June 1-July 24). Partners contributing hospitalizations only were in California (June 23-August 4), Colorado (June 3July 20), Minnesota and Wisconsin (July 1-August 2), and New York (June 30-July 25).

** Full vaccination was defined as index testing or admission date $\geq 14$ days after second dose of BNT162b2 (Pfizer-BioNTech) or mRNA-1273 (Moderna) or after a single dose of Ad26.COV2 (Janssen) vaccines.

the months before Delta became predominant $(2,4)$. However, VE against COVID-19 hospitalization among adults aged $\geq 75$ years was significantly lower than that among adults aged $<75$ years, which had not been observed previously from this data source (4). This moderate decline should be interpreted with caution and might be related to changes in SARS-CoV-2, waning of vaccine-induced immunity with increased time since vaccination, or a combination of factors. Differences in VE between the two mRNA vaccines, which had not been observed previously in the VISION Network (4), are consistent with another recent finding. ${ }^{\$}$ Further examination of the magnitude and sources of product-specific VE differences are also warranted.

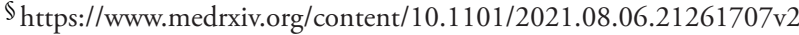


The findings in this report are subject to at least three limitations. First, VE by time since vaccination was not examined; further evaluation of possible waning of vaccine protection is currently underway. Second, VE for partial vaccination was not assessed. Finally, although the facilities in this study serve heterogenous populations in nine states, the findings might not be generalizable to the U.S. population.

These findings reaffirm the high protection of COVID-19 vaccines against moderate and severe COVID-19 resulting in $\mathrm{ED}, \mathrm{UC}$, and hospital visits and underscore the importance of full COVID-19 vaccination and continued benefits of COVID-19 vaccination during Delta variant predominance.

\section{Acknowledgments}

Catherine H. Bozio, Sue Reynolds, Jill Ferdinands, Palak Patel, Eric P. Griggs, Rachael M. Porter, Lenee Blanton, Andrea Steffens, Natalie Olson, Stephanie J. Schrag, Jennifer R. Verani, Alicia M. Fry, Eduardo Azziz-Baumgartner, CDC COVID-19 Response Team; Kristin Dascomb, Nancy Grisel, Julie Arndorfer, Division of Infectious Diseases and Clinical Epidemiology, Intermountain Healthcare; Brian E. Dixon, William F. Fadel, Nimish Ramesh Valvi, Peter J. Embi, Center for Biomedical Informatics, Regenstrief Institute; Sarah W. Ball, Rebecca J. Birch, Matthew E. Levy, Maria Demarco, Yan Zhuang, Patricia Shifflett, Westat; Stephanie A. Irving, Center for Health Research, Kaiser Permanente Northwest; Suchitra Rao, Michelle A. Barron, Department of Medicine, University of Colorado; Elyse O. Kharbanda, HealthPartners Institute; Jungmi Han, Department of Biomedical Informatics, Columbia University; Ned Lewis, Kristin Goddard, Bruce Fireman, Ousseny Zerbo, Kaiser Permanente Vaccine Study Center, Kaiser Permanente Northern California; Anupam Kharbanda, Children's Minnesota; Manjusha Gaglani, Baylor Scott \& White Health, Texas A\&M University College of Medicine.

Corresponding author: Mark G. Thompson, isq8@cdc.gov, for the VISION Network.

${ }^{1}$ Center for Biomedical Informatics, Regenstrief Institute, Indianapolis, Indiana;

${ }^{2}$ Westat, Rockville, Maryland; ${ }^{3}$ Department of Medicine, University of Colorado, Anschutz Medical Campus, Aurora, Colorado; ${ }^{4}$ Division of Infectious Diseases and Clinical Epidemiology, Intermountain Healthcare, Salt Lake City, Utah; ${ }^{5}$ Kaiser Permanente Vaccine Study Center, Kaiser Permanente Northern California, Oakland, California; ${ }^{6}$ HealthPartners Institute, Minneapolis, Minnesota; ${ }^{7}$ Center for Health Research, Kaiser Permanente Northwest, Portland, Oregon; ${ }^{8}$ Department of Biomedical Informatics, Columbia University, New York, New York; ${ }^{9}$ CDC COVID-19 Response Team.
All authors have completed and submitted the International Committee of Medical Journal Editors form for disclosure of potential conflicts of interest. Shaun J. Grannis reports grants from the Patient-Centered Outcomes Research Institute, Agency for Healthcare Research and Quality, National Institute of Mental Health, National Center for Advancing Translational Sciences, and California Healthcare Foundation; consulting fees from RTI International and Indiana Health Information Exchange; and two U.S. patent applications unrelated to this publication: "Method and system for creating synthetic unstructured free tax medical data for training machine learning models" (\#20200035360) and "Predictive Modeling For Health Services" (\#20200312457). Nicola P. Klein reports research support from Pfizer for COVID-19 vaccine clinical trials and research support from Pfizer, Merck, GlaxoSmithKline, Sanofi Pasteur, and Protein Sciences (now Sanofi Pasteur) for unrelated studies. Allison L. Naleway reports funding from Vir Biotechnology for research unrelated to this study and Pfizer research funding to Kaiser Permanente Northwest for unrelated study of meningococcal $\mathrm{B}$ vaccine safety during pregnancy. No other potential conflicts of interest were disclosed.

\section{References}

1. Thompson MG, Burgess JL, Naleway AL, et al. Prevention and attenuation of Covid-19 with the BNT162b2 and mRNA-1273 vaccines. N Engl J Med 2021;385:320-9. PMID:34192428 https://doi. org/10.1056/NEJMoa2107058

2. Tenforde MW, Self WH, Naioti EA, et al.; IVY Network Investigators; Ivy Network. Sustained effectiveness of Pfizer-BioNTech and Moderna vaccines against COVID-19 associated hospitalizations among adultsUnited States March-July 2021. MMWR Morb Mortal Wkly Rep 2021;70:1156-62. PMID:34437524 https://doi.org/10.15585/mmwr. $\mathrm{mm} 7034 \mathrm{e} 2$

3. Haas EJ, Angulo FJ, McLaughlin JM, et al. Impact and effectiveness of mRNA BNT162b2 vaccine against SARS-CoV-2 infections and COVID-19 cases, hospitalisations, and deaths following a nationwide vaccination campaign in Israel: an observational study using national surveillance data. Lancet 2021;397:1819-29. PMID:33964222 https:// doi.org/10.1016/S0140-6736(21)00947-8

4. Thompson MG, Stenehjem E, Grannis S, et al. Effectiveness of COVID-19 vaccines in ambulatory and inpatient care settings. $\mathrm{N}$ Engl J Med 2021 Epub September 8, 2021. PMID:34496194 https://doi. org/10.1056/NEJMoa2110362 